\title{
Thickness of Compressed Hair Layer: A Pilot Study in a Manikin
}

\author{
Stijn VERWULGEN ${ }^{*}$, Jochen VLEUGELS ${ }^{1}$, Daniël LACKO ${ }^{1}$, \\ Erik HARING ${ }^{1}$, Guido DE BRUYNE ${ }^{1}$, Toon HUYSMANS ${ }^{2}$ \\ ${ }^{1}$ Department of Product Development, Fac. of Design Sciences, University of Antwerp, Belgium; \\ 2 iMinds-Vision Lab, Dept. of Physics, University of Antwerp, Belgium \\ DOI: 10.15221/16.182 http://dx.doi.org/10.15221/16.182
}

\begin{abstract}
Recent advancements in 3D anthropometry enable to link a subject's $1 \mathrm{D}$ measurements to its $3 \mathrm{D}$ shape to achieve better fit, functionality, comfort and/or safety. Statistical shape models of the human head retrieved from medical images (CT or MRI) allow for such parameterized models, with a recently proven accuracy of $1.6 \mathrm{~mm}$ with only four scalp parameters. This induces opportunities for better sizing systems and mass customization of head mounted products. Due to the nature of medical images, hair is never present in these models. Head shapes constructed from conventional optical 3D scans however do capture the presence of hair. In current optical 3D scans, subjects wear a swimming cap, hairnet or wig cap to flatten and compress the hair layer and prevent artifacts, interferences and misinterpretations during scanning due to the presence of hair. Thus models based on medical images provide parameterized scalp models with proven accuracy. This might be required for the design of certain head mounted wearables were sensors/actuators should make proper contact with the scalp through the hair. However, many other products to be designed for closely fitting the human head will rest on a (flattened) layer of hair. Thus in these situations it might be required to take account of the flattened and compressed hair layer in the design process. Up till now, knowledge of the compressed hair layer geometry is rarely needed in the field of industrial design with only anecdotic numbers available. The uprising of accurate parameter driven 3D models of the human head and entailed applications will induce the need for further accurate quantification thereof.

Firstly, we present two a method to assess the thickness of the flattened and compressed hair layer by capturing hair thickness at a given point with a linear dial gauge. Secondly, we present another method to quantify the hair layer from points measured on scalp and hair layer. From this geometric information one can also deduce whether and to what extend the parametric scalp model approximates the head model with hair layer with the same accuracy as it approximates the scalp.

Effect of hair thickness was evaluated by measuring a manikin with and without wigs and caps that flattened and compressed the wigs. All experiments were thus conducted in vitro, but both methods can be transferred without burdens to in vivo experiments.

A hair thickness between $1.3 \pm 0.4 \mathrm{~mm}$ was observed with the first method and $1.5 \pm 1.3 \mathrm{~mm}$ with the second method. A small number of measurements indicate that the head form with flattened and compressed hair is predicted by the parametric scalp model with the same accuracy as it predicts the scalp form.

These pilot results indicated that for the design of head mounted products that rest on the flattened hair layer, a margin of at least $1.5 \mathrm{~mm}$ should be taken into account for eventual variations in hair thickness. For the design of (personalized) head mounted products, already established parameterized scalp models can be used without loss of accuracy towards the presence of a flattened hair layer. Further large scale and in vivo studies are required to confirm and fine-tune these results.
\end{abstract}

Keywords: shape modeling, compressed hair layer, 3D anthropometry, scalp, industrial design

\section{Introduction}

Human sizes and shapes exhibit many variations. Linear -1D- anthropometrical measurements and their statistical distribution are used to link body dimensions to product dimensions and to design products accordingly for a target population. For the design of products that ought to fit closely on the human body, 1D anthropometrical measurements alone are insufficient to achieve proper fit, comfort and/or functionality, as shown for the design of helmets were accurate and representative 3D models of the human head are desired [1]. Advancements in 3D anthropometry have induced the possibility to predict a subject's true 3D body shape from a set of 1D anthropometrical measurements as constrains provided to a statistical shape model of the body or body part as prior knowledge [2-4]. A statistical shape model is a collection of 3D forms such that each point on a given form has a corresponding 'similar' point on any other form of the collection [5]. Advancements in 3D scanning, image

* corresponding author: stijn.verwulgen@uantwerpen.be 
reconstruction and image processing resulted in a realm of databases containing accurate 3D shape information of the human body [6, 7], targeting the full -3D- human shape variation through statistical shape models. The availability of statistical shape models and the techniques to predict any subject specific 3D model from it by providing anthropometric measurements, allows for the parameterization of a subjects 3D body or body part thereof. Parameterized models can be used as a basis for design equations. This technique potentially results in better sizing systems and eventually even also driving personalized production techniques because the same design can be tuned to any individual body surface and e.g. 3D printed accordingly [6].

For the design of some categories of head mounted wearables such as hearing aids or EEG headsets, sensors/actuators should make proper contact with the scalp through the hair. Thus predictive models of the head and scalp are required, discarding the eventual presence of hair. Up till now, the hair layer geometry has not drawn much of attention in the field of industrial design but the uprising of accurate parameter driven 3D models of the human head $[8,9]$ will induce the need for accurate quantification of the hair layer. For the design of many other categories of future head mounted products such as (personalized/improved) helmets and other protective products, the product will rest on a (flattened) layer of hair. Thus accurate predictive models of the head will be required, taking account of eventual presence of (flattened) hair.

\subsection{Predictive models for the head and scalp}

With data from the international consortium for brain mapping (IBCM)-[10], a statistical shape model of the human scalp was constructed. It was shown that a (pseudo-) random selection of $100 \mathrm{MRI}$ scans suffice to construct a statistical shape model of the human scalp that represents any other scalp of that database with and accuracy of $1 \mathrm{~mm}^{3}$ [8]. It was shown that only four easy to measure anthropometrical measurements: head length $(H L)$, bitragion width $(B W)$, head circumference $(H C)$ and arc length $(A L)$ of the head predict the scalp with average spatial accuracy of $1.6 \mathrm{~mm}$ and standard deviation of 0.4 $\mathrm{mm}$ (Fig. 1, see also [8] p.74).

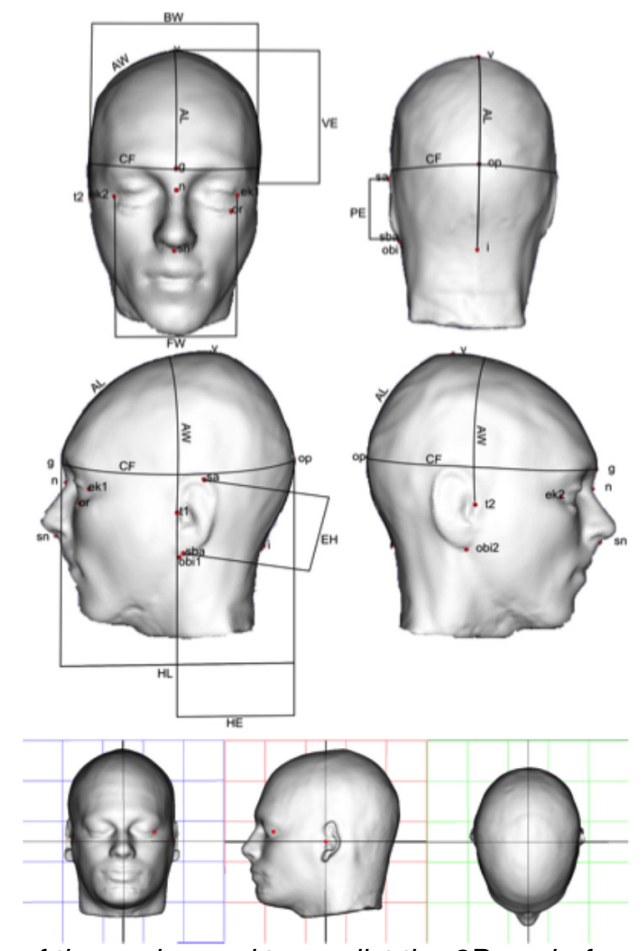

Fig. 1. Anatomical measurements of the scalp used to predict the $3 D$ scalp form: head length (HL), bitragion width $(B W)$, head circumference $(H C)$ and arc length $(A L)$ of the head and respective anatomical landmarks, taken from

[8]. As well as reference points $n$ and $t(1-2)$ to align the heads.

These results are retrieved from digital models in which correspondence is group-wise optimized to achieve maximal compactness [11]. Thereby, the working hypothesis is that the scalp models retrieved from the MRI database is representative for the target population of Caucasian ethnicity of male as well as females between 20 and 40 years. Partial and indirect verification of this hypothesis was conducted through anthropometric assessment of real world subjects [3]. Scalp parameters HL, BW, $\mathrm{HC}$ and $\mathrm{AL}$ were measured and taken as parameters in the model of [8] to predict a subject's 3D scalp. 
A 3D model of the subjects' scalp was constructed by interpolating 39 points measured onto the scalp with a Microscribe 3D Digitizer probe through an EEG cap. Predicted and constructed scalp models were compared, yielding an average prediction error of $2 \mathrm{~mm}$. This is only slightly bigger than the 1.6 $\mathrm{mm}$ obtained in [8]. Differences might be explained by instability of measurement of surface points, e.g. induced by the presence of hair. Due to the nature of medical images (CT or MRI), hair was never present in the scans on which the shape model was based.

\subsection{Models for the head with compressed hair layer}

In current optical 3D scans, subjects wear a swimming cap, hairnet or wig cap to compress the hair and prevent artifacts, interferences and misinterpretations during image constructing due to the presence of hair [12]. Skull models constructed from conventional 3D scans are complementary to those constructed from medical scans (CT or MRI), in the sense that the former delimit the outer border of the external form from optical registration, and the latter delimit the inner border of the external form from internal information. The difference between both is the compressed hair layer. Thus for a (non bald) subject, the corresponding scalp model is only approximated due to the presence of hair layer beneath the cap.

\subsection{Hair layer geometry}

Knowledge of the compressed hair layer geometry is rarely applied in the field of industrial design with only anecdotic numbers available. We are only aware of applications in heat models of the skull to design heat-exchanging products i.e. a cold cap for prevention of hair loss during chemotherapy for cancer treatment. An offset between 1 and $2 \mathrm{~mm}$ is introduced in these studies $[13,14]$ however without further references to scientific research to support these values. Hair simulation has been a long standing challenge in computer animation [15] with mathematical equations for wisp-based simulations to produce realistic looking simulation already established for more than a decade [16] and real time simulation methods established recently [17]. However, none of these computer models have been verified to yield accurate simulations of the flattened hair layer e.g. when wearing a helmet. The link between computer animations and predictive models that can be used for the purpose of industrial design requires cautious verification of ground truth of these models, which requires assessment of hair in various conditions.

\subsection{Research questions}

The parametric models constructed and studied in [8] are lacking hair, making the prediction especially suitable for applications in which a precise contact with the scalp is required. However if the predicted shape of the head is close enough to the shape of the head with hair, the models can also be used with consideration to represent a non-bald head. It would be relevant to know the geometry of the compressed hair layer: 1) to know how big an offset should be added to average and subject specific scalp/head models retrieved from medical/optical 3D images to accommodate for specific types and volume/length of hairs, 2) to know how the compressed hair layer is positioned around the subject-specific parameterized model of the scalp from medical images, whether these models also predict the scalp comprising the compressed hair layer within its accuracy of $1.6 \mathrm{~mm}$, or whether similar models need to be constructed from optical images, to that end.

We present and compare two methods to assess the hair layer on manikins, that can be easily transferred to an in vivo setting. The second method will also allow us to evaluate how hair layer is distributed around the parametric scalp models constructed in [8].

\section{Materials and methods}

The thickness of the flattened hair layer compressed by a wig cap is assessed by capturing hair thickness at a given point with a linear dial gauge. The 3D coordinates of points at the scalp are captured to estimating a subject's scalp by a surface interpolating various 3D measured points. We show how surface points on both scalp and compressed hair layer can be used to compare relative position of the hair layer around the parameterized scalp model to quantify whether and to what extend that model also approximates the hair layer with the same accuracy as it approximates the scalp. Both methods are applied in small sized settings by measuring manikins with and without wigs and caps that flattens and compress the wigs. Three wigs that are used in theatre were selected to reflect a variety of hair types and volumes. Wigs are placed on a gypsum 3D printed model of human head for subsequent measurements: on compressed hair layer and scalp surface with and without hair layer. 
The head model is an average from the statistical shape model in [8], thus each individual model representing the subject's head and scalp, without hair due to the nature of the underlying medical images. A standard EEG cap is placed each time on each wig, flattening and compressing the artificial hair onto the head model. The cap contains markers of 39 points based on the 10-20 location-system as used in EEG signal captation [18], yielding uniformly distributed annotations on the head.

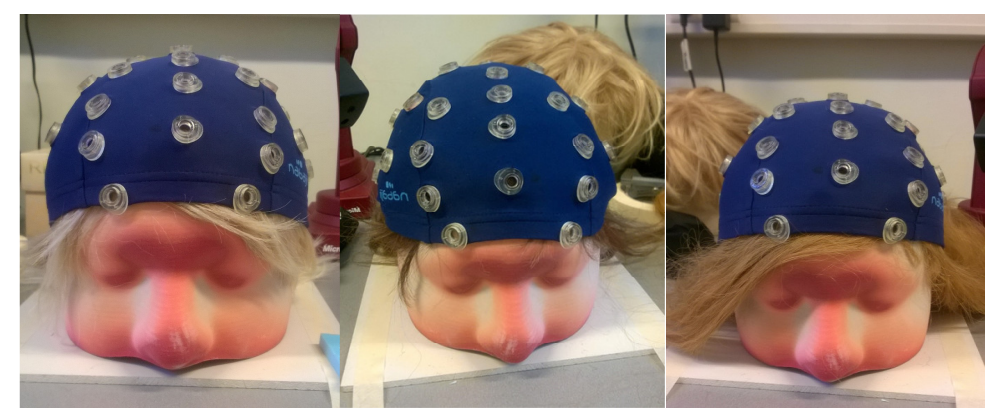

Fig. 2. The three different wings with the measurement cap placed on the $3 D$ printed head.

\subsection{Compressed hair layer thickness assessment}

\subsubsection{Equipment}

Each point in the 10-20 location-system is marked by a plastic ring onto the textile cap with an offset/height of $6.5 \mathrm{~mm}$ (Fig. 3. yellow part), perpendicular on the compressed hair and scalp surface. The dial gauge has a shaft with protruding pen, both with radial symmetry. The pen has a fixed protruding distance in neutral position from which any further protrusion displacement is generated by exerting a slight force. Displacement is displayed on the dial with an accuracy of $0.01 \mathrm{~mm}$. The measurement range of the dial gauge is $10 \mathrm{~mm}$, allowing to the targeted measurement range of about $2 \mathrm{~mm}$ learned from estimates in previous research [13]. A support part with radial symmetry was designed for close fitting the shaft (Fig. 3. green part). The design was 3D printed with ABS and encloses the shaft without gliding, only removable up and down under a force far bigger than the force required to generate further displacement of the pen. The purpose of the support part is thus threefold: 1) to position and control the depth of the pen such that it outer end is precisely at the outer surface of the hair layer in neutral position, 2) to create a surface to intercept counter force for further displacement of the pen through the (wig) hair layer onto the (gypsum) scalp surface, 3 ) to ensure that the pen penetrates the hair layer perpendicular by positioning the outer surface of the support part onto the 10-20 point marker, which is done automatically correct by the mild force required for penetrating the hair layer and thus further displacement of the gauge pen. Fig. 3 shows how the hair layer thickness $\mathrm{X}$ is measured with the dial gauge.
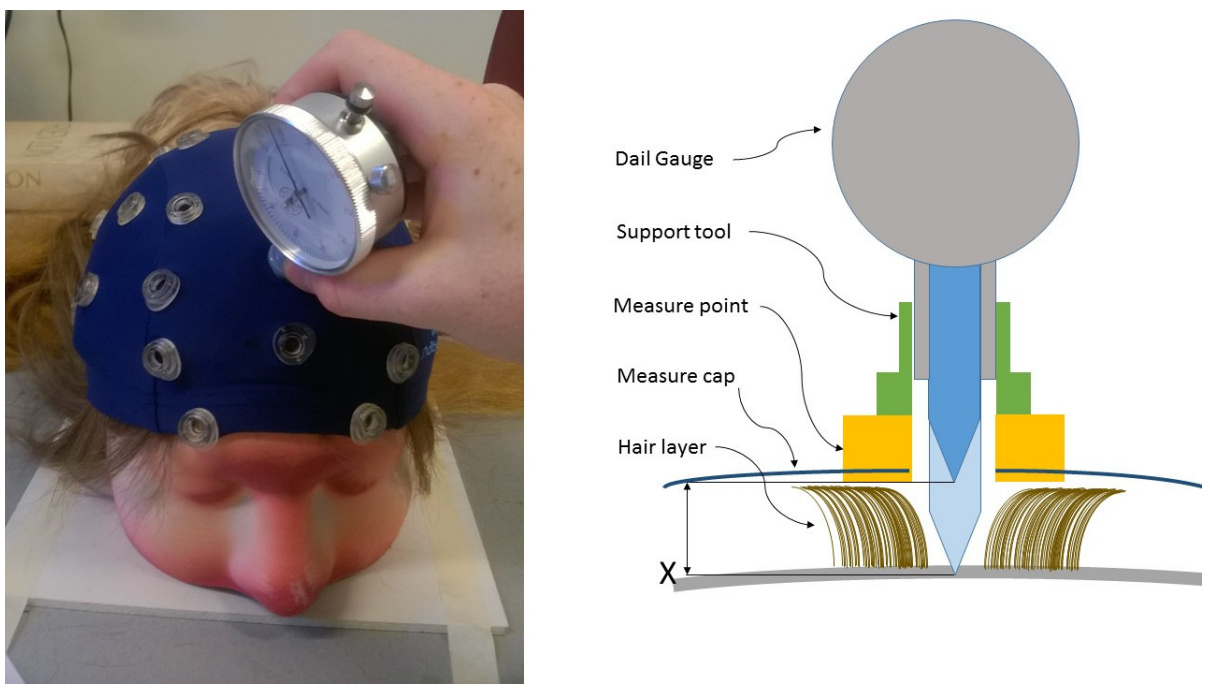

Fig. 3. Left a picture of the measurement with a dial gauge on the 3D printed head with wig and measurement cap. Right a schematic representation of the measurement with the dial gauge. 


\subsubsection{Measurements}

The 3D printed human head model is fixated to eliminate eventual movement during and/or due to measuring. Hair thickness is measured at four points: two points in the sagital plane at most dorsal and ventral positions and two points in the frontal plane at most lateral positions. The support part on the dial gauge is placed at the markers and the maximum distance the gauge pen can travel is measured (distance $X$ in Fig. 3). On each wig, set of measurements is conducted three times and an average is taken. Values at all points and for all wigs are averaged.

\subsection{Parameterized model for scalp with compressed hair layer}

\subsubsection{Equipment}

The four basic anthropometric measurements $\mathrm{HL}, \mathrm{BW}, \mathrm{HC}$ and $\mathrm{AL}$ as defined in Fig. 1 are collected on the 3D printed gypsum model with an outside caliper and a tape measure. The 3D location of points at the scalp and surface of compressed hair layer are recorded with a Microscribe 3D digitizing device. This device records at every button press and has an accuracy of up to $0.05 \mathrm{~mm}$. The EEG cap provides 39 reference points from the 10-20 system marked by rings that have a hole so that the measuring probe can measure trough these markers. Measurement equipment is shown in Fig. 4.

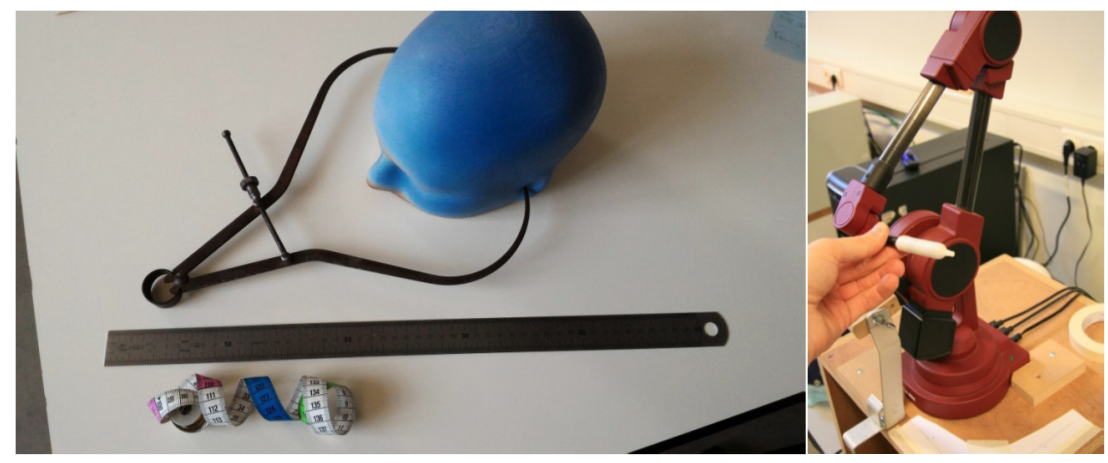

Fig. 4. Measuring setup with outside caliber, ruler and tape measure and Microscribe digitizer.

The fact that the surface of the gypsum 3D printed head is known can be used as a ground truth to assess the accuracy of the Microscribe 3D recordings. Accuracy is a necessary condition to transfer this method to an in vivo setting.

\subsubsection{Measurements}

After a wig is mounted on the head model, it is a first time flattened and compressed with the EEG cap. Then the 3D coordinates of all 39 points in the 10-20 reference system are collected, as well as both tragi and nasion as reference points for future alignments. The measuring pen of the Microscribe is relatively small $(4 \mathrm{~mm}$ in diameter, $20 \mathrm{~mm}$ long and with a pointy tip) such that points can be effectively measured through the hair on the skin. The EEG cap is removed and the wig is a second time flattened and compressed by a stretch swimming cap mounted on the wig. Then the EEG cap is remounted on top of the swimming cap. The same 3 reference locations and the 39 annotations of the cap are measured again, yielding a second set of 42 locations. This time the Microscribe Digitizer pen reaches the surface of the swimming cap instead of probing through the hair up to the scalp. Thus the pen stops during registration on the hair layer (with the thickness of the swimming cap) and not the skin. Each wig is measured three times with and without swimming cap and resulting positions are averaged. Thus for each wig, we have two sets of 42 reference points: one of the scalp and one of the compressed hair layer. Note that, a pair of points of the same marker, one on the scalp and one on the hair layer, will generally not be aligned perpendicular to the surface of the scalp, due to repositioning of the EEG cap after remounting it on the wig with swimming cap. Thus point-to-point distance will generally by an erroneous estimate of hair layer.

\subsubsection{Parameterized scalp model for skull with compressed hair layer: an accuracy metric}

A custom script was written, in a combination of Python for the data processing and PHP for visualization and data collecting, to conduct the actions described below.

All data is collected in a PHP script (web-interface internally available) where all 1D data on anthropometric parameters can be entered and the 3D data could be uploaded. A tool automatically process the 1D data and subsequently, the collected measurements are used as an input to predict the 
best matching 3D scalp shape by the statistical shape model constructed in [9] using a Python script. The result is an .stl file with 10000 vertices. The predicted stl.-model is aligned with each of the two sets of 42 measured points by first a rough alignment based on the 3 reference points taken in each measurement (this aligned the model within $2^{\circ}$ rotational and $2 \mathrm{~mm}$ movement accuracy) followed by an iterative closest point method for further optimization of the alignment. Thereby the sum of squared distances of each of the measured points to its closest point on the stl model is minimized. Alignment towards both scalp and swimcap points is needed to evaluate how hair layer is positioned around the parametric scalp model.

To calculate the distance between a pair of points marking the scalp and hair surface, one should anticipate a shift of the EEG cap after repositioning. To that end, one could implement a method whereby the closest distance from a measuring point to the predicted head shape is calculated along perpendicular projection, calculated a first time for the point on the hair layer and a second time for a point on the scalp, after which both distances are added. This estimate of the thickness of the hair layer is more accurate than the (generally longer) Euclidean distance between both measuring points. We modify this method to a signed distance to obtain a metric that estimates whether and to what extend the predicted head shape also models the head with flattened hair layer. To that end, the distance of a point at the hair layer to the predicted head model is signed negative if that point lies at the interior, and positive if that point lies at the exterior of the predicted head model. Reverse signing convention is used for points on the scalp. Adding both signed distances of hair and scalp points at a given EEG marker yields a signed distance. The absolute value of that signed distances represents the thickness of the hair layer at that marker. The average of signed distances reflects to what extent point at hair layer and scalp are equally well positioned at both orientations (inside and outside) of the predicted head model.

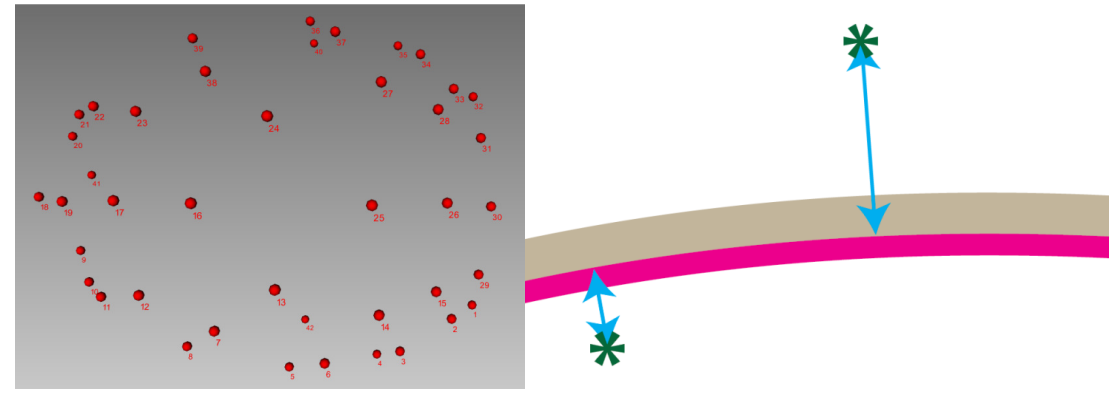

Fig. 5. Top view of all the points measured off the 10-20 reference cap; points 40 to 42 are the three reference locations (left) and orientation of a pair of points and estimate of hair layer, with pink zone marking the inside and grey zone marking the outside of the skull model (right).

\section{Results}

All measurements were conducted by the fourth-named author along the same protocol for maximal repeatability and to control intra-operator errors.

A hair thickness of $1.3 \mathrm{~mm}$ with a standard deviation of $0.4 \mathrm{~mm}$ was obtained by the dial gauge measurement method. Repeated measurements at same points with the same wigs are within the accuracy of the dial gauge $0.01 \mathrm{~mm}$. An outlier hair thickness of $2.23 \mathrm{~mm}$ was observed at some point. The measurements were easy and intuitive to conduct and robust with no missing data.

The thickness of the swimming cap also measured by the dial gauge was around $0.3 \mathrm{~mm}$.

In was shown that the Microscribe digitizer assessed the group of 42 points of interest on the head with an accuracy of $0.5 \mathrm{~mm}$. This was done by registering these 42 points on the gypsum model through the EEG cap, aligning the known surface of the gypsum model with these measured points and calculate the average distance as a measure for accuracy.

The measurements to obtain averaged signed distance of hair layer was performed by collecting points at the 42 markers with the Microscribe digitizer. Each collection of 42 points was done three times with the intention to take averages to flatten out eventual occasional variation in pressed depth. So each wig was assessed 6 times: 3 times with flattened and compressed wig hair layer and 3 times without compressing swimming cap through the wig hair layer onto the (wig) scalp. Out of the 18 data sets of the measurement cap only 9 contained the complete set of 42 data points and only one matching pair, indicating that this method was not robust even conducted by the same operator. Nonetheless, the data was usable to calculate the hair layer thickness. The average signed hair layer thickness of the test was $-0.07 \mathrm{~mm}$ with a standard deviation of $1.14 \mathrm{~mm}$. The averaged hair thickness was $1.5 \mathrm{~mm}$ with a standard deviation of $.1 .3 \mathrm{~mm}$. 


\section{Discussion}

Thickness of flattened hair can be easily measured with an EEG cap and a dial gauge equipped with a support part. First results indicate that this hair layer is fairly consistent with regard to measuring point and hair type. Moreover a thickness value of $1.3 \mathrm{~mm}$ found in these experiments confirms earlier preliminary estimates of between $1 \mathrm{~mm}$ and $2 \mathrm{~mm}[13,14]$. The measurements with the dial gauge also indicate that the hair layer thickness can enlarge the size of the head up to $2.2 \mathrm{~mm}$. As only four points are measured, more measuring points at the head are required to refine these results. Thereby the EEG cap has a double function: annotating points at which the hair thickness is measured along the 10-20 system and flattening and compressing the hair layer. This double function has the advantage that it requires no remounting after mounting a flexible swimming cap or hairnet used in conventional 3D scanning to flatten and compress the hair. All real world interpretations of results are under the assumption that the theatre wigs are a correct representation of real human hair. Due to the easy set up and short measuring time (a few seconds), this method is easy to conduct in in-vivo settings. The method could be adapted to measure the effect of compressing the hair layer by other type of flexible headwear, modifying that headwear by marks along the 10-20 system and a 3D support system on the dial gauge that fits these marks.

An averaged signed distance of -0.07 found in the second experiment indicate that the hair layer is equally spread along the inside and outside of the predicted head model The amount of incomplete data sets indicates that the method to measure the 42 different measurement points with the Microscribe is however not robust and not feasible. During the test and after each measurement, the data set should be checked if all the 42 coordinate points are in place. However this can considerably increase the time to complete the measurements due to the amount of measurements that needs to be redone to complete the measuring set. More complete set of measurements on scalp and hair layer are needed to refine these results. The average hair layer thickness of $1.5 \mathrm{~mm}$ derived from these measurements is in line with the thickness of $1.3 \mathrm{~mm}$ found with the dial gauge but the standard deviation of $1.3 \mathrm{~mm}$ is much larger. This might be explained by the limited accuracy of $0.5 \mathrm{~mm}$ by which the Microscribe can assess the group of 42 points of interest. Also, if the proposed measurement method is used in an in-vivo setting, errors caused by the slight movement of the head might further decrease accuracy.

The found thickness of $1.3 \mathrm{~mm}$ found in the first experiments is within the $1.6 \mathrm{~mm}$ accuracy boundary of the predicted head model [8]. Combined with the averaged signed distance of $-0.07 \mathrm{~mm}$ found in the second experiment might lead to the conclusion that a design on the parameter driven head model that takes account of a parameterization error of at least $1.6 \mathrm{~mm}$, will equally well fit bold and non-bold subjects. Thus the presence of hair might be neglected in that setting. However if more accurate parameterized models become available (e.g. by increasing the number of parameters) with accuracy around or exceeding the thickness of hair layer, an offset should be taken into account at the zones were hair grows, to model scalp with hair layer.

Systematic and large-scale studies in human subjects are required to pinpoint thickness of compressed hair layer and confirm these results, potentially only for specific hair types. The dimensions and physiology of individual scalp hair is well known [19] but thickness of compressed hair layer will be highly related to physical characteristics. Thereby, sample size for systematic studies will be greatly affected by hair type due to worldwide variation and classification of human hair [20,21]. In such program, a map should be constructed between hair layer thickness and hair type to be assessed by objective and simple measurements to know how to design close fitting head mounted products for multiple groups with design guidelines in function of hair type rather than ethnicity [22].

\section{Conclusion}

Two methods to assess subject's flattened and compressed hair layer were proposed, by simulating subjects' hair layer condition as in optical 3D body scanning. Both methods were tested by placing wigs and EEG cap on a 3D printed head model. For the first method, only a dial gauge and support part towards EEG cap was needed to assess thickness of hair layer, but only thickness and no geometric information of the hair layer was thus gathered. This test setting yielded stable and reproducible measurements with and estimated average thickness of $1.3 \mathrm{~mm}$ with a standard deviation of $0.4 \mathrm{~mm}$ along different measure points and different wigs. The second method took account of geometric position of both scalp and hair layer and yielded an avaerage hair thickness of $1.5 \mathrm{~mm}$ with a standard deviation of $1.3 \mathrm{~mm}$. These methods can be easily transferred to an in-vivo setting and with headwear alternative to EEG caps to flatten and compress the hair while taking a 3D body scan.

We also compared the geometric position of the hair layer around a parameter driven predictive head model. Pilot tests with such a model of the scalp retrieved from medical images indicate that both hair 
and scalp layer are evenly distributed at the inside and outside of the predictive model. The hair layer of $1.3-1.5 \mathrm{~mm}$ is absorbed by the parameterization error of $1.6 \mathrm{~mm}$. These pilot results indicated that for the design of head mounted products that rest on the flattened hair layer, a margin of about $1.5 \mathrm{~mm}$ should be taken into account for eventual variations in hair thickness. For the design of (personalized) head mounted products, the tested parameter driven predictive head model can be used without loss of accuracy towards the presence of a flattened hair layer. Further large scale and in vivo studies are required to confirm and fine-tune these results.

\section{References}

[1] P. Meunier, D. Tack, A. Ricci, L. Bossi, and H. Angel, "Helmet accommodation analysis using 3D laser scanning," Applied Ergonomics, vol. 31, pp. 361-369, 2000.

[2] S.-Y. Baek and K. Lee, "Parametric human body shape modeling framework for human-centered product design," Computer-Aided Design, vol. 44, pp. 56-67, 2012.

[3] J. Vleugels, D. Lacko, G. De Bruyne, T. Huysmans, and S. Verwulgen, "Physical evaluation of an anthropometric shape model of the human scalp," in Proceedings of the 3D Body Scanning Technology conference, 2015, doi:10.15221/15.161.

[4] F. DANCKAERS, T. HUYSMANS, D. LACKO, and J. SIJBERS, "Evaluation of 3D Body Shape Predictions Based on Features," in 6th International Conference on 3D Body Scanning Technologies, Lugano, Switzerland 2015, pp. 258-265, doi:10.15221/15.258.

[5] I. L. Dryden and K. V. Mardia, Statistical shape analysis vol. 4: J. Wiley Chichester, 1998.

[6] S. Verwulgen, D. Lacko, G. De Bruyne, F. Danckaers, N. Christis, J. Sijbers, et al., "Anthropometrics 2.0: Enrichment of Classical Anthropometry through Multidisciplinary Collaboration," in DS 78: Proceedings of the E\&PDE 2014 16th International conference on Engineering and Product Design, University of Twente, The Netherlands, 2014.

[7] R. TRIEB, A. BALlester, G. KARTSOUNIS, S. ALEMANY, J. URIEL, G. HANSEN, et al., "EUROFIT-Integration, Homogenisation and Extension of the Scope of Large 3D Anthropometric Data Pools for Product Development," in 4th International Conference on 3D Body Scanning Technologies, Long Beach, CA, USA, 2013, doi:10.15221/13.258.

[8] D. Lacko, T. Huysmans, P. M. Parizel, G. De Bruyne, S. Verwulgen, M. M. Van Hulle, et al., "Evaluation of an anthropometric shape model of the human scalp," Applied ergonomics, vol. 48, pp. 70-85, 2015.

[9] D. Lacko, T. Huysmans, S. Verwulgen, P. M. Parizel, M. M. Van Hulle, and J. Sijbers, "Statistical shape modelling in support of user-centred $\mathrm{BCl}$ headset design."

[10] J. C. Mazziotta, A. W. Toga, A. Evans, P. Fox, and J. Lancaster, "A probabilistic atlas of the human brain: theory and rationale for its development the international consortium for brain mapping (ICBM)," Neuroimage, vol. 2, pp. 89-101, 1995.

[11] T. Huysmans, J. Sijbers, and V. Brigitte, "Automatic construction of correspondences for tubular surfaces," IEEE transactions on pattern analysis and machine intelligence, vol. 32, pp. 636-651, 2010.

[12] R. Ball, "3-D design tools from the SizeChina project," Ergonomics in Design, vol. 17, pp. 8-13, 2009.

[13] A. Frijns, G. Van Leeuwen, and A. Van Steenhoven, "Modelling heat transfer in humans," ERCOFTAC Bull, vol. 68, pp. 43-47, 2006.

[14] M. Sheikholeslami, M. Ghaffari, A. Khorasani, and M. Zoghi, "Site-Dependence Scalp Cooling System to Prevent Hair Loss during Chemotherapy," Journal of Bioengineering \& Biomedical Sciences, vol. 5, p. 1, 2015.

[15] K. Ward, F. Bertails, T.-Y. Kim, S. R. Marschner, M.-P. Cani, and M. C. Lin, "A survey on hair modeling: Styling, simulation, and rendering," IEEE Transactions on Visualization and Computer Graphics, vol. 13, pp. 213-234, 2007.

[16] E. Plante, M.-P. Cani, and P. Poulin, "Capturing the complexity of hair motion," Graphical Models, vol. 64, pp. 40-58, 2002.

[17] K. Wu and C. Yuksel, "Real-time hair mesh simulation," in Proceedings of the 20th ACM SIGGRAPH Symposium on Interactive 3D Graphics and Games, 2016, pp. 59-64.

[18] R. W. Homan, J. Herman, and P. Purdy, "Cerebral location of international 10-20 system electrode placement," Electroencephalography and clinical neurophysiology, vol. 66, pp. 376-382, 1987.

[19] B. Buffoli, F. Rinaldi, M. Labanca, E. Sorbellini, A. Trink, E. Guanziroli, et al., "The human hair: from anatomy to physiology," International journal of dermatology, vol. 53, pp. 331-341, 2014.

[20] R. De La Mettrie, D. Saint-Léger, G. Loussouarn, A. Garcel, C. Porter, and A. Langaney, "Shape variability and classification of human hair: a worldwide approach," Human biology, vol. 79, pp. 265-281, 2007.

[21] N. Otberg, "Ethnic variation in hair," Textbook of Cosmetic Dermatology, p. 386, 2010.

[22] G. Loussouarn, A. L. Garcel, I. Lozano, C. Collaudin, C. Porter, S. Panhard, et al., "Worldwide diversity of hair curliness: a new method of assessment," International journal of dermatology, vol. 46, pp. 2-6, 2007. 